\title{
ON THE DIMENSION OF LEFT INVARIANT MEANS AND LEFT THICK SUBSETS
}

BY

\author{
MARIA KLAWE
}

\begin{abstract}
If $S$ is a left amenable semigroup, let $\operatorname{dim}\langle M l(S)\rangle$ denote the dimension of the set of left invariant means on $S$. THEOREM. If $S$ is left amenable, then $\operatorname{dim}\langle M l(S)\rangle=n\langle\infty$ if and only if $S$ contains exactly $n$ disjoint finite left ideal groups. This result was proved by Granirer for $S$ countable or left cancellative. Moreover, when $S$ is infinite, left amenable, and either left or right cancellative, we show that $\operatorname{dim}\langle M l(S)\rangle$ is at least the cardinality of $S$. An application of these results shows that the radical of the second conjugate algebra of $l_{1}(S)$ is infinite dimensional when $S$ is a left amenable semigroup which does not contain a finite ideal.
\end{abstract}

1. Introduction. Let $S$ be a discrete semigroup, and $m(S)$ the Banach space of bounded real-valued functions with the sup norm. An element $\mu \in m(S)^{*}$ is called a mean if $\|\mu\|=1$ and $\mu(f) \geqslant 0$ for $f \geqslant 0$. For each $s \in S$, let $l_{s}\left[r_{s}\right]$ denote the linear operator on $m(S)$ defined by $l_{s} f(t)=f(s t)\left[r_{s} f(t)=f(t s)\right]$ for $t \in S$, and $f \in m(S)$. A mean $\mu$ is said to be left [right] invariant if $\mu\left(l_{s} f\right)=\mu(f)\left[\mu\left(r_{s} f\right)=\mu(f)\right]$ for each $f \in m(S)$ and each $s \in S$. Let $M l(S)$ $[M r(S)]$ denote the set of left [right] invariant means on $S$, and $\langle M l(S)\rangle$ $[\langle M r(S)\rangle]$ denote its linear span in $m(S)^{*}$. When $S$ has a left [right] invariant mean, we say $S$ is left [right] amenable.

It is natural to look for conditions which determine the dimension of $\langle M l(S)\rangle$ when $S$ is left amenable. The first results in this direction were given by Day [5, p. 535], who proved that infinite solvable groups, infinite amenable nontorsion groups, and infinite locally finite groups, all have more than one left invariant mean. In [12, p. 43], Luthar showed that a commutative semigroup has a unique left invariant mean if and only if it has a finite ideal. Continuing the search for conditions that $\langle M l(S)\rangle$ be finite dimensional, Granirer [7, p. 32] proved the following theorem:

THEOREM (GRANIRER). If $S$ is a countably infinite left amenable semigroup,

Received by the editors February 9, 1976.

AMS (MOS) subject classifications (1970). Primary 43A07; Secondary 20M10, 16A21, 43A05, 28A70.

Key words and phrases. Semigroup, left thick, invariant means, radical, finite left ideal group.

- American Mathematical Society 1977 
then $\operatorname{dim}\langle M l(S)\rangle=n\langle\infty$ if and only if $S$ contains exactly $n$ disjoint finite groups which are left ideals $\left(^{(}\right)$.

Except for the case where $S$ is left cancellative [7, p. 49], Granirer was unable to drop the countability condition; he was only able to replace it by a slightly weaker one (see [7, p. 44]).

When $S$ is an infinite left amenable semigroup with cancellation, Chou has proved [2] that $\operatorname{dim}\langle M l(S)\rangle \geqslant 2^{\varpi} \cdot|S|$, where $\sqrt{5}$ denotes the cardinality of the continuum, and $|S|$ the cardinality of $S$. Later Chou [3], using an idea of Kakutani and Oxtoby in $[11, \S 16]$, was able to prove that $\operatorname{dim}\langle M l(S)\rangle=2^{2^{|S|}}$ when $S$ is an infinite amenable group.

The purpose of this paper is to investigate the structure of the left thick subsets of infinite left amenable semigroups, and use this to obtain lower bounds for the dimension of the set of left invariant means. By this method, we are able to prove Granirer's theorem without the countability condition, and hence prove Luthar's result for all left amenable semigroups. Another easily obtained corollary is that if $S$ is amenable (both left and right) and $\operatorname{dim}\langle M l(S)\rangle$ is finite, then $\operatorname{dim}\langle M l(S)\rangle=\operatorname{dim}\langle M r(S)\rangle=1$ (i.e. $S$ has a unique invariant mean). This generalizes another theorem of Granirer [8, Theorem 1] who proved the above proposition under the additional hypothesis that $\operatorname{dim}\langle\operatorname{Mr}(S)\rangle$ be finite. Our techniques also yield that if $S$ is either right cancellative or left cancellative as well as infinite and left amenable, then $\operatorname{dim}\langle M l(S)\rangle \geqslant|S|$.

In $\$ 2$ we define the terms left thickness and strong left thickness for subsets of $S$, and illustrate their connection with $\operatorname{dim}\langle M l(S)\rangle$ via Remark 2.1 and Theorem 2.2, which deals with the existence of "large" collections of pairwise disjoint left thick subsets of $S$. This section concludes with some specific results for the right cancellative case.

In $\$ 3$ we define uniform left thickness, and give an equivalent characterization in terms of the behavior of left invariant means on the characteristic functions of subsets of $S$ with smaller cardinality than $S$ (Proposition 3.1).

Our main result giving a lower bound for $\operatorname{dim}\langle M l(S)\rangle$ is found in $\S 4$ (Theorem 4.1), as well as the generalizations of the theorems of Granirer and Luthar (Theorem 4.4, Corollaries 4.5 and 4.6).

$\S 5$ gives an application of the results of $\S 4$ to the radical of the second conjugate algebra $m(S)^{*}$. In $\S 6$ we look at thickness properties for the left

(')In Granirer's original theorem [7, p. 32], the condition " $S$ contains exactly $n$ disjoint finite groups which are left ideals" was stated as " $S$ contains exactly $n$ disjoint finite left ideal left cancellative groups" (a set $A \subset S$ is said to be left ideal left cancellative if $A$ is a left ideal in $S$, and whenever $s a=s b$ for $s \in S$ and $a, b \in A$, then $a=b$ ). However, as Granirer later noted, if $G$ is a finite group with identity $e$, and also a left ideal in $S$, then for each $s \in S$ we have $s G=s(e G)=(s e) G=G$, which implies that $G$ is left ideal left cancellative. 
cancellative case, while $\S 7$ is devoted to examples. $\$ 8$ considers the limitations involved in using our techniques to find the exact size of $M l(S)$.

The results of this paper will form part of my thesis under the direction of Anthony T. Lau, to whom I am very grateful for suggesting this problem, and who also pointed out several of the applications of the main theorems.

2. Strong left thickness and collections of pairwise disjoint left thick subsets. Let $S$ be a semigroup with the discrete topology. For each subset $A \subset S$, let $\chi_{A}$ denote the characteristic function of $A$ (for each $s \in S, \chi_{A}(s)=1$ if $s \in A$ and $\chi_{A}(s)=0$ if $\left.s \notin A\right)$, and let $|A|$ denote the cardinality of $A$. We say that $A$ is left thick in $S$ if for each finite subset $F \subset S$ we can find $s \in S$ with Fs $\subset A$. In [13, Theorem 7], Mitchell showed that a subset $A$ of a left amenable semigroup $S$ is left thick if and only if $\mu\left(\chi_{A}\right)=1$ for some $\mu \in M l(S)$.

We say that $A \subset S$ is strongly left thick if for each $B \subset S$ with $|B|<|A|$, the set $A \backslash B$ is left thick in $S$. Although every semigroup is obviously left thick in itself, it is easy to find examples of left amenable semigroups which are not strongly left thick. Consider finite groups for instance, or left amenable semigroups which contain a right ideal of smaller cardinality. An example of this last type is given in $\S 7$ (Example 7.3).

RemarK 2.1. If $\left\{A_{\gamma}: \gamma \in \Gamma\right\}$ is a collection of pairwise disjoint left thick subsets of a left amenable semigroup $S$, then for each $\gamma \in \Gamma$ we can choose $\mu_{\gamma} \in M l(S)$ with $\mu_{\gamma}\left(\chi_{A_{\gamma}}\right)=1$. This set $\left\{\mu_{\gamma}: \gamma \in \Gamma\right\}$ of left invariant means on $S$ is linearly independent. In fact if $\sum_{i=1}^{n} a_{i} \mu_{i}=0$ for some $\left\{\mu_{1}, \ldots, \mu_{n}\right\} \subset$ $\left\{\mu_{\gamma}: \gamma \in \Gamma\right\}$, then for $j=1, \ldots, n$ we have $a_{j}=\sum_{i=1}^{n} a_{i} \mu_{i}\left(\chi_{A_{j}}\right)=0$. Thus we are interested in finding collections of pairwise disjoint left thick subsets of $S$.

The following theorem shows that strong left thickness is a useful property in determining lower bounds for the dimension of $\langle M l(S)\rangle$.

THEOREM 2.2. $A$ subset $A \subset S$ is strongly left thick if and only if there exists a collection $\left\{D_{\gamma}: \gamma \in \Gamma\right\}$ of pairwise disjoint subsets of $A$, which are left thick in $S$, such that $|\Gamma|=|A|$.

Proof. Suppose $A$ is strongly left thick. If $A$ is finite, then for each $a \in A$ we have $\{a\}=A \backslash(A \backslash\{a\})$ is left thick in $S$; hence $\{\{a\}: a \in A\}$ is the desired collection. Thus we may assume $A$ is infinite.

Let $\omega$ be the first ordinal with $|\omega| \equiv|\{\alpha: \alpha<\omega\}|=|A|$. Write $A=\left\{a_{\alpha}\right.$ : $\alpha<\omega\}$ and let $A_{\beta}=\left\{a_{\alpha}: \alpha<\beta\right\}$ if $1 \leqslant \beta<\omega$. Using transfinite induction we construct a family $\left\{t_{(\beta, F)}: 1 \leqslant \beta<\omega, F\right.$ a finite subset of $\left.A_{\beta}\right\}$ with the following properties:

(i) $F t_{(\beta, F)} \subset A$ for each finite $F \subset A_{\beta}$, where $1 \leqslant \beta<\omega$.

(ii) Let $X_{\beta}=\cup\left\{F t_{(\beta, F)}\right.$ : finite $\left.F \subset A_{\beta}\right\}$ if $1 \leqslant \beta<\omega$. Then $X_{\beta} \cap X_{\delta}=$ $\varnothing$ for $1 \leqslant \beta<\delta<\omega$. 
$A_{1}=\left\{a_{0}\right\}$ and since $A$ is left thick, there exists $t \in S$ such that $a_{0} t \in A$. Let $t_{\left(1,\left\{a_{0}\right\}\right)}=t$.

Suppose we have constructed a family $\left\{t_{(\beta, F)}: 1 \leqslant \beta<\delta, F\right.$ finite $\left.\subset A_{\beta}\right\}$ satisfying (i) and (ii), where $1<\delta<\omega$. Let $Y=\cup\left\{X_{\beta}: 1 \leqslant \beta<\delta\right\}$, and let $|\delta|$ denote $|\{\alpha: \alpha<\delta\}|$. If $|\delta|$ is finite, then $Y$ is a finite union of finite sets so $|Y|<|A|$. If $|\delta|$ is infinite, then $\left|X_{\beta}\right| \leqslant|\delta|$ for $1 \leqslant \beta<\delta$, and we have $|Y| \leqslant|\delta|^{2}=|\delta|<|A|$, since $\delta<\omega$. Thus $|Y|<|A|$ in either case, hence $A \backslash Y$ is left thick, and so for each finite subset $F \subset A_{\delta}$ we can choose $t_{(\delta, F)} \in S$ such that $F t_{(\delta, F)} \subset A \backslash Y$. Then $X_{\delta} \subset A \backslash Y$ and $X_{\beta} \cap X_{\delta}=\varnothing$ for $1 \leqslant \beta<\delta$.

Let $\Gamma=\{\gamma: 1 \leqslant \gamma<\omega\}$. Choose a $1-1$ correspondence $T: \Gamma \rightarrow \Gamma \times \Gamma$ and define $\Gamma_{\gamma}=T^{-1}(\Gamma \times\{\gamma\})$ for each $\gamma \in \Gamma$. Now each $\Gamma_{\gamma}$ is cofinal in $\Gamma$ since $\left|\Gamma_{\gamma}\right|=|\Gamma|$, and if $\gamma_{1} \neq \gamma_{2}$ then $\Gamma_{\gamma_{1}} \cap \Gamma_{\gamma_{2}}=\varnothing$.

For each $\gamma \in \Gamma$, define $D_{\gamma}=\cup\left\{X_{\beta}: \beta \in \Gamma_{\gamma}\right\}$. Clearly $\left\{D_{\gamma}: \gamma \in \Gamma\right\}$ is a collection of pairwise disjoint subsets of $A$. Since $|\Gamma|=|\omega|=|A|$, it only remains to show that each $D_{\gamma}$ is left thick in $S$. Since $A$ is left thick in $S$, it suffices to show that $D_{\gamma}$ is left thick in $A$. Let $F$ be a finite subset of $A$. Then for some $\beta$, we must have $F \subset A_{\beta}$, and since $\Gamma_{\gamma}$ is cofinal in $\Gamma$ we may assume $\beta \in \Gamma_{\gamma}$. Now $F t_{(\beta, F)} \subset X_{\beta} \subset D_{\gamma}$, and we see that $D_{\gamma}$ is left thick.

Conversely, let $\left\{D_{\gamma}: \gamma \in \Gamma\right\}$ be a collection of pairwise disjoint subsets of $A$, which are left thick in $S$, such that $|\Gamma|=|A|$. Suppose $B \subset S$ with $|B|<|A|$. Since $|\Gamma|>|B|$, there exists $\gamma \in \Gamma$ with $B \cap D_{\gamma}=\varnothing$. Then $D_{\gamma} \subset$ $A \backslash B$ so $A \backslash B$ is left thick, which finishes the proof.

Next we show that every infinite left amenable semigroup with right cancellation is strongly left thick. In $\$ 6$, we will see that this remains true when right cancellation is replaced by left cancellation. First we need the following lemmas:

LEMMA 2.3. If $B$ and $C$ are subsets of an infinite semigroup $S$ with right cancellation such that $|B|<|S|$ and $|C|<|S|$, then there exists $s \in S$ with $B \cap s C=\varnothing$.

Proof. If not, then for each $s \in S$ we can find a pair $\left(b_{s}, c_{s}\right) \in B \times C$ such that $b_{s}=s c_{s}$. If $s \neq t$, then $\left(b_{s}, c_{s}\right) \neq\left(b_{t}, c_{t}\right)$ since otherwise we would have $s c_{s}=b_{s}=b_{t}=t c_{t}=t c_{s}$, and hence $s=t$ by right cancellation. Thus $|B \times C|=|B||C| \geqslant|S|$, which contradicts $|B|<|S|$ and $|C|<|S|$ since $S$ is infinite.

LEMMA 2.4. If $B$ is a subset of an infinite semigroup $S$ with right cancellation such that $|B|<|S|$, then there exists a sequence $\left\{s_{n}\right\} \subset S$ with $s_{n} B \cap s_{m} B=$ $\varnothing$ for $n \neq m$.

Proof. Construct the sequence $\left\{s_{n}\right\}$ by induction. Choose $s_{1} \in S$ arbi- 
trarily. If we have constructed $s_{1}, \ldots, s_{n}$ such that $s_{i} B \cap s_{j} B=\varnothing$ for $1 \leqslant i<$ $j \leqslant n$, then by Lemma 2.3 we can find $s_{n+1} \in S$ so that $s_{n+1} B \cap\left(s_{1} B\right.$ $\left.\cup \cdots \cup s_{n} E\right)=\varnothing$, since $\left|s_{1} B \cup \cdots \cup s_{n} B\right|<|S|$.

Proposition 2.5. If $S$ is an infinite left amenable semigroup with right cancellation, then for each $B \subset S$ with $|B|<|S|$ and for each $\mu \in M l(S)$ we have $\mu\left(\chi_{B}\right)=0$.

Proof. By Lemma 2.4 there exists a sequence $\left\{s_{n}\right\} \subset S$ with $s_{n} B \cap s_{m} B=$ $\varnothing$ for $n \neq m$. Thus $1=\mu\left(\chi_{S}\right) \geqslant \sum_{n=1}^{\infty} \mu\left(\chi_{s_{n} B}\right)$. For each $n$ we have $l_{s_{n}} \chi_{s_{n} B} \geqslant$ $\chi_{B}$ and so $\mu\left(\chi_{s_{n} B}\right)=\mu\left(l_{s_{n}} \chi_{s_{n} B}\right) \geqslant \mu\left(\chi_{B}\right)$. Hence $\mu\left(\chi_{B}\right)=0$.

COROLlaRY 2.6. If $S$ is an infinite left amenable semigroup with right cancellation, then every left thick subset $A$ is strongly left thick. In particular $S$ is strongly left thick.

Proof. Choose $\mu \in M l(S)$ so that $\mu\left(\chi_{A}\right)=1$. If $B \subset S$ with $|B|<|A|$, then $\mu\left(\chi_{(A \backslash B)}\right) \geqslant \mu\left(\chi_{A}\right)-\mu\left(\chi_{B}\right)=1$. Thus $A \backslash B$ is left thick.

3. Uniform left thickness. In this section we examine another thickness property for semigroups. We say $S$ is uniformly left thick if $|A|=|S|$ for every left thick subset $A \subset S$. As a corollary to the next proposition, we see that for infinite left amenable semigroups, uniform left thickness implies strong left thickness.

Proposition 3.1. An infinite left amenable semigroup $S$ is uniformly left thick if and only if for each $B \subset S$ with $|B|<|S|$ and for each $\mu \in M l(S)$ we have $\mu\left(\chi_{B}\right)=0$.

Before giving the proof we introduce a few necessary definitions and notation. A relation $R$ is defined on a semigroup $S$ by $a R b$ for $a, b \in S$, if there exists $u \in S$ with $a u=b u$. If the intersection of finitely many right ideals of $S$ is always nonempty (as is the case when $S$ is left amenable), then $R$ is an equivalence relation, the set $S^{\prime}$ of equivalence classes is a semigroup with right cancellation under the induced multiplication, and the quotient map $\pi: S \rightarrow S^{\prime}$ is a semigroup homomorphism. For more details, see Granirer [8, p. 371].

Proof of Proposition 3.1. Suppose $S$ is uniformly left thick, $B \subset S$ with $|B|<|S|$, and $\mu \in M l(S)$. If $\left|S^{\prime}\right|=|S|$, define $\mu^{\prime} \in M l\left(S^{\prime}\right)$ by $\mu^{\prime}(f)=$ $\mu(f \circ \pi)$ for each $f \in m\left(S^{\prime}\right)$. Then $|\pi(B)|<\left|S^{\prime}\right|$, so by Proposition 2.5 we have $0=\mu^{\prime}\left(\chi_{\pi(B)}\right)=\mu\left(\chi_{\pi(B)} \circ \pi\right) \geqslant \mu\left(\chi_{B}\right) \geqslant 0$.

Thus we may assume $\left|S^{\prime}\right|<|S|$. For each $g \in S^{\prime}$ choose $t_{g} \in \pi^{-1}(g)$. Let $C$ be the subset of $S$ defined as $\left\{t_{g} b: g \in S^{\prime}, b \in B\right\}$. Since $S$ is infinite, $|C|<|S|$. If $\mu\left(\chi_{B}\right)>0$, then $C$ is left thick in $S$. To see this, we first show that for any finite set $F \subset S$, there exists $u \in S$ such that $F u=\left\{t_{g} u\right.$ : 
$g \in \pi(F)\}$. It is easily seen by induction on $|F|$, that for each $g \in \pi(F)$ there exists $u_{g} \in S$ with $\left(F \cap \pi^{-1}(g)\right) u_{g}=t_{g} u_{g}$. Now choose $u \in \bigcap_{g \in \pi(F)} u_{g} S$. Then $F u=\left\{t_{g} u: g \in \pi(F)\right\}$. If $\mu\left(\chi_{B}\right)>0$, then $B \cap u S \neq \varnothing$ since $\mu\left(\chi_{u s}\right)=$ 1. Therefore we can find $s \in S$ so that $u s \in B$. But now Fus $=\left\{t_{g} u s\right.$ : $g \in \pi(F)\} \subset C$. Hence $C$ is left thick. Since $S$ is uniformly left thick and $|C|<|S|$, we must have $\mu\left(\chi_{B}\right)=0$.

Conversely, if for each $B \subset S$ with $|B|<|S|$ we have $\mu\left(\chi_{B}\right)=0$ for all $\mu \in M l(S)$, then clearly $B$ is not left thick in $S$.

COROLlaRY 3.2. For infinite left amenable semigroups, uniform left thickness implies strong left thickness.

The proof is identical to that of Corollary 2.6.

COROLlaRY 3.3. Every infinite left amenable semigroup with right cancellation is uniformly left thick.

This follows immediately from Proposition 2.5.

REMARK 3.4. The above proposition fails when we allow $S$ to be finite. Suppose $S$ is a finite group. Then its only left thick subset is the group itself; so $S$ is uniformly left thick. However, if $\mu$ is the unique left invariant mean on $S$, then $\mu\left(\chi_{B}\right)=|B||S|^{-1} \neq 0$ if $B \neq \varnothing$.

In $\$ 7$ we give an example of an infinite left amenable semigroup which is strongly left thick but not uniformly left thick. Since most of the proof of Proposition 3.1 is devoted to the case where $\left|S^{\prime}\right|<|S|$, an example of a uniformly left thick infinite left amenable semigroup $S$ with $\left|S^{\prime}\right|<|S|$ is also given in \$7. In view of Proposition 3.1, it is interesting to note a similar reformulation of strong left thickness for left amenable semigroups.

Proposition 3.5. A left amenable semigroup is strongly left thick if and only if for each $B \subset S$ with $|B|<|S|$ there exists $\mu \in M l(S)$ with $\mu\left(\chi_{B}\right)=0$.

4. A lower bound for $\operatorname{dim}\langle M l(S)\rangle$. We define the width of a semigroup $S$, denoted by $W(S)$, as $W(S)=\sup \{|A|: A$ is a strongly left thick subset of $S\}$. Now we are ready to prove our main result.

THEOREM 4.1. If $S$ is a left amenable semigroup which contains no finite left thick subsets, then $\operatorname{dim}\langle M l(S)\rangle \geqslant W(S) \geqslant \aleph_{0}$.

Proof. That $\operatorname{dim}\langle M l(S)\rangle \geqslant W(S)$ follows immediately from Theorem 2.2 and Remark 2.1. Thus we need only show that $S$ contains an infinite strongly left thick subset. Let $A$ be a left thick subsemigroup of $S$ so that $|A|$ is minimal. Clearly $A$ is infinite, and also uniformly thick since if $B \subset A$ is left thick in $A$ with $|B|<|A|$, then the subsemigroup generated by $B$ is left thick in $S$, and has cardinality less than $A$. Since $A$ is left thick in $S$, the semigroup 
$A$ is left amenable. Thus by Corollary 3.2, $A$ is strongly left thick.

REMARK 4.2. Even when $S$ contains no finite left thick subsets, we may have $W(S)<|S|$ (see Example 7.3).

With the help of this theorem, we proceed to give a proof of Granirer's theorem without the countability condition, and then the generalization of Luthar's result, as mentioned in $\S 1$. For this we need one more lemma.

We use the term left ideal group to signify a group which is also a left ideal in $S$.

LEMMA 4.3. If a left amenable semigroup $S$ contains a finite left thick subset, then $S$ contains a finite left ideal group.

Proof. Suppose $A \subset S$ is a finite left thick subset. The left ideal $S a$ is finite for some $a \in A$, since $\mu\left(\chi_{A}\right)=1$ for some $\mu \in M l(S)$, implies $\mu\left(\chi_{\{a\}}\right)>0$ for some $a \in A$. Choose $t \in S$ such that $S t$ is finite, with $|S t|$ minimal, and let $C=S t$. It is easy to check that $C$ is right cancellative, and since $C$ is also finite and left amenable, $C$ is a finite group.

THEOREM 4.4. A left amenable semigroup has $\operatorname{dim}\langle M l(S)\rangle=n\langle\infty$ if and only if $S$ contains exactly $n$ disjoint finite left ideal groups.

Proof. In [7, p. 34], Granirer proved that if $S$ contains exactly $n$ disjoint finite left ideal groups, then $\operatorname{dim}\langle M l(S)\rangle=n$. Now suppose $\operatorname{dim}\langle M l(S)\rangle=$ $n<\infty$. The semigroup $S$ must contain some finite left ideal group since otherwise Lemma 4.2 and Theorem 4.1 show that $\operatorname{dim}\langle M l(S)\rangle$ is not finite. $S$ cannot contain infinitely many disjoint finite left ideal groups, since by Remark 2.1 that would again imply that $\operatorname{dim}\langle M l(S)\rangle$ is infinite. Thus $S$ contains exactly $m$ disjoint finite left ideal groups for some finite number $m$, and by Granirer's result, we see $m=n$.

COROLlaRY 4.5. If $S$ is a left amenable semigroup, then $\operatorname{dim}\langle M l(S)\rangle$ is finite if and only if $S$ contains a finite two-sided ideal.

Proof. If $\operatorname{dim}\langle M l(S)\rangle=n<\infty$, then $S$ contains exactly $n$ disjoint finite left ideal groups, say $A_{1}, \ldots, A_{n}$. In [7, p. 34], Granirer shows that $\cup\left\{A_{i}\right.$ : $i=1, \ldots, n\}$ is a finite two-sided ideal.

If $A$ is a finite two-sided ideal of $S$, then for each $\mu \in M l(S)$ we have $\mu\left(\chi_{A}\right)=1$, and hence $\operatorname{dim}\langle M l(S)\rangle=\operatorname{dim}\langle M l(A)\rangle$, which is finite since $A$ is finite.

COROLlaRY 4.6. If $S$ is an amenable (both left and right) semigroup with $\operatorname{dim}\langle M l(S)\rangle=n\langle\infty$, then $S$ contains a finite group which is a two-sided ideal, and hence $\operatorname{dim}\langle M l(S)\rangle=\operatorname{dim}\langle M r(S)\rangle=1$ (i.e. $S$ has a unique invariant mean). 
Proof. As shown in Corollary 4.5, $S$ contains a finite two-sided ideal $A$ which is the union of $n$ disjoint finite left ideal groups. By footnote 1 (p. ) we see that $A$ is left cancellative. Since $A$ is also right amenable and finite, $A$ is a finite group. Let $\mu \in m(S)^{*}$ be defined as $\mu(f)=|A|^{-1} \Sigma\{f(a): a \in A\}$. Then $\operatorname{Ml}(S)=\operatorname{Mr}(S)=\{\mu\}$.

Theorem 4.4 is proved by Granirer for $S$ countable [7, p. 32], or $S$ left cancellative [7, p. 49]. Moreover, he proved Corollary 4.6 for these cases [7, p. 46], and also for the general case under the additional assumption that $\operatorname{dim}\langle\operatorname{Mr}(S)\rangle$ be finite [8, Theorem 1]. Corollary 4.5 was proved by Luthar [12, p. 43] for $S$ commutative.

5. An application to the radical of the second conjugate algebra $m(S)^{*}$. Let $l_{1}(S)$ be the space of real-valued functions $\theta$ on $S$ such that $\Sigma\{|\theta(s)|: s \in S\}$ is finite. For $\nu \in m(S)^{*}$ and $f \in m(S)$, we define $\nu * f \in m(S)$ by $\nu * f(s)$ $=\nu\left(l_{s} f\right)$. Now for $\mu, \nu \in m(S)^{*}$, the Arens multiplication on $m(S)^{*}$ is defined as $\mu * \nu(f)=\mu(\nu * f)$ for each $f \in m(S)$. Under this multiplication $m(S)^{*}$ becomes the second conjugate algebra of $l_{1}(S)$ (for more details see Day [5, p. 526]).

THEOREM 5.1. If $S$ is a left amenable semigroup which contains no finite ideals, then the radical $J$ of $m(S)^{*}$ is infinite dimensional.

Proof. Using an idea of Civin and Yood (see [4, pp. 849-850]), it can be shown that $M l(S) \subset J+\mu$ for any $\mu \in M l(S)$. Since $M l(S)$ is infinite dimensional by Corollary 4.5 , the radical $J$ must be also.

Granirer used the same proof to show that the radical of $m(S)^{*}$ is infinite dimensional when $S$ is a commutative semigroup without finite ideals (see [8, p. 378]), and also when $S$ is an infinite left amenable group (see [7, p. 48]).

COROLlaRY 5.2. Let $S$ be a left amenable semigroup and suppose the radical of $m(S)^{*}$ is finite dimensional. If $S$ is either left cancellative or right cancellative, then $S$ is finite.

Proof. By Theorem 5.1, $S$ contains a finite ideal $A$. Now for any $a \in A$, we have $a S \cup S a \subset A$. If $S$ is either left cancellative or right cancellative, then $|S| \leqslant|a S \cup S a| \leqslant|A|$, and we see that $S$ is finite.

REMARK. This result is already known for the left cancellative case (Granirer and Rajagopalan [10]). It is unknown whether it still holds when the cancellative properties of $S$ are dropped. However, the following example shows that the condition that $S$ is left amenable and contains a finite ideal is not enough to ensure that the radical of $m(S)^{*}$ is finite dimensional.

EXAMPLE 5.3. Let $S=\left\{s_{n}: n=0,1,2, \ldots\right\}$ with the multiplication $s_{i} s_{j}=$ $s_{0}$ for all $i, j$. Then $\mu \in m(S)^{*}$, defined by $\mu(f)=f\left(s_{0}\right)$ for each $f \in m(S)$, is the unique left (and right) invariant mean on $S$, and $\left\{s_{0}\right\}$ is a finite ideal in $S$. 
For each $n \geqslant 1$, define $\phi_{n} \in m(S)^{*}$ by $\phi_{n}(f)=f\left(s_{n+1}\right)-f\left(s_{n}\right)$ for each $f \in m(S)$. Now the set $\left\{\phi_{n}: n=1,2, \ldots\right\}$ is linearly independent in $m(S)^{*}$, and for any $\nu \in m(S)^{*}$ we have $\nu * \phi_{n}=\phi_{n} * \nu=0$ for each $n$. Thus $\left\{\phi_{n}\right.$ : $n=1,2, \ldots\} \subset J$ and, hence, $J$ is infinite dimensional.

A further illustration that the radical $J$ of $m(S)^{*}$ may be much larger than the ideal $A=\left\{\mu_{1}-\mu_{2}: \mu_{1}, \mu_{2} \in M l(S)\right\}$ is given by Civin and Yood in [4, Theorem 3.5], where they show that $J / A$ is infinite dimensional when $S$ is the additive group of integers.

6. Thickness properties for infinite left amenable semigroups with left cancellation. The purpose of this section is to show that every infinite left amenable semigroup with left cancellation is strongly left thick. In order to do this, we first prove some preliminary lemmas.

LEMMA 6.1. If $B$ and $C$ are subsets of an infinite semigroup $S$ with left cancellation, such that $|B|<|S|$ and $|C|<|S|$, then there exists $s \in S$ with $B \cap C s=\varnothing$.

Proof. Replace right cancellation by left cancellation in the proof of Lemma 2.3.

Given $s \in S$ and $\mu \in M l(S)$, define $\mu_{(s)} \in m(S)^{*}$ by $\mu_{(s)}(f)=\mu\left(r_{s} f\right)$ for each $f \in m(S)$, where $r_{s} f(t)=f(t s)$ for $t \in S$. It is easily checked that $\mu_{(s)} \in M l(S)$, and that for each $A \subset S$, we have $\mu_{(s)}\left(\chi_{A s}\right) \geqslant \mu\left(\chi_{A}\right)$.

LEMMA 6.2. If $S$ is an infinite left amenable semigroup with left cancellation and $B \subset S$ with $|B|<|S|$, then there exists a sequence $\left\{\mu_{n}\right\} \subset M l(S)$ such that $\sum_{n=1}^{\infty} \mu_{n}\left(\chi_{B}\right) \leqslant 1$.

Proof. Using induction, we construct sequences $\left\{\mu_{1}, \mu_{2}, \ldots\right\} \subset M l(S)$ and $\left\{s_{2}, s_{3}, \ldots\right\} \subset S$ with the following property:

$$
\begin{aligned}
\mu_{n}\left(\chi\left(B \cup B s_{n} \cup B s_{n-1} s_{n} \cup \cdots \cup B s_{2} s_{3} \cdots s_{n}\right)\right. & \\
& \geqslant \mu_{n}\left(\chi_{B}\right)+\mu_{n-1}\left(\chi_{B}\right)+\cdots+\mu_{1}\left(\chi_{B}\right) .
\end{aligned}
$$

Clearly this sequence $\left\{\mu_{n}\right\}$ satisfies the statement of the lemma, since (i) implies that $1=\mu_{n}\left(\chi_{S}\right) \geqslant \mu_{n}\left(\chi_{B}\right)+\mu_{n-1}\left(\chi_{B}\right)+\cdots+\mu_{1}\left(\chi_{B}\right)$ for each $n$. Choose $\mu_{1} \in M l(S)$ arbitrarily. By Lemma 6.1 we can find $s_{2} \in S$ with $B \cap B s_{2}=\varnothing$. Let $\mu_{2}=\mu_{1\left(s_{2}\right)}$ as defined above.

If we have constructed $\left\{\mu_{1}, \ldots, \mu_{n-1}\right\}$ and $\left\{s_{2}, \ldots, s_{n-1}\right\}$ for some $n>3$, then by Lemma 6.1 we can find $s_{n}$ with

$$
B \cap\left(B \cup B s_{n} \cup B s_{n-1} \cup \cdots \cup B s_{2} s_{3} \cdots s_{n-1}\right) s_{n}=\varnothing .
$$

Let $\mu_{n}=\mu_{n-1\left(s_{n}\right)}$. Then we have 


$$
\begin{aligned}
& \mu_{n}\left(\chi\left(B \cup B s_{n} \cup B s_{n-1} s_{n} \cup \cdots \cup B s_{2} s_{3} \cdots s_{n}\right)\right) \\
&=\mu_{n}\left(\chi_{B}\right)+\mu_{n}\left(\chi\left(B \cup B s_{n-1} \cup B s_{n-2} s_{n-1} \cup \cdots \cup B s_{2} s_{3} \cdots s_{n-1}\right) s_{n}\right) \\
& \geqslant \mu_{n}\left(\chi_{B}\right)+\mu_{n-1}\left(\chi\left(B \cup B s_{n-1} \cup B s_{n-2} s_{n-1} \cup \cdots \cup B s_{2} s_{3} \cdots s_{n-1}\right)\right) \\
& \geqslant \mu_{n}\left(\chi_{B}\right)+\mu_{n-1}\left(\chi_{B}\right)+\mu_{n-2}\left(\chi_{B}\right)+\cdots+\mu_{1}\left(\chi_{B}\right) .
\end{aligned}
$$

Proposition 6.3. If $S$ is an infinite left amenable semigroup with left cancellation, then $S$ is strongly left thick.

Proof. Let $B \subset S$ with $|B|<|S|$. By Lemma 6.2 we can find a sequence $\left\{\mu_{n}\right\} \subset M l(S)$ with $\sum_{n=1}^{\infty} \mu_{n}\left(\chi_{B}\right) \leqslant 1$. Clearly we must have $\lim _{n \rightarrow \infty} \mu_{n}\left(\chi_{B}\right)=$ 0 . Since $M l(S)$ is weak*-compact, we can find a subnet $\left(\mu_{\alpha}\right)$ of $\left\{\mu_{n}\right\}$ which is weak*-convergent to $\mu \in M l(S)$. Then $\mu\left(\chi_{B}\right)=0$, so $\mu\left(\chi_{(S \backslash B)}\right)=1$ and hence $S \backslash B$ is left thick in $S$.

REMARK. Although every infinite left amenable semigroup with right cancellation is uniformly left thick, this does not remain true when right cancellation is replaced by left cancellation (see Example 7.4).

7. Examples. The first three examples are special cases of the following general type of left amenable semigroup. If $S$ is a lattice (a partially ordered set in which every finite set has a supremum and an infimum), then a multiplication can be defined on $S$ by $a b=\sup \{a, b\}$ for $a, b \in S$. This operation is commutative and associative and, hence, $S$ is a left amenable semigroup ( $S$ is even extremely left amenable, i.e. $m(S)$ admits a multiplicative left invariant mean, see [9]). We call this operation the sup multiplication. A subset $A \subset S$ is left thick in $S$ if and only if $A$ is cofinal in $S$.

EXAMPLE 7.1. A left amenable semigroup containing no finite left thick subsets, which is strongly left thick but not uniformly left thick.

Let $S$ be the real numbers under the sup multiplication. The set of integers is left thick in $S$; hence $S$ is not uniformly left thick. If $B \subset S$ with $S \backslash B$ not left thick, then the interval $[a, \infty)$ is contained in $B$ for some $a \in S$, and we have $|B|=|S|$. Thus $S$ is strongly left thick.

EXAMPLE 7.2. An infinite uniformly left thick left amenable semigroup $S$ which is extremely left amenable. In particular, $\left|S^{\prime}\right|<|S|$.

Let $S$ be the integers under the sup multiplication. Obviously $S$ is uniformly left thick since every cofinal subset is infinite. Also $\left|S^{\prime}\right|=1$ since for each $a, b \in S$ there exists $c \in S$ with $a c=b c$.

EXAMPLE 7.3. A left amenable semigroup containing no finite left thick subsets, which is not strongly left thick.

Let $S$ be the union of the interval $[0,1]$ with the natural numbers $\mathbf{N}$ under the sup multiplication. $S$ has no finite left thick subsets since every cofinal subset is infinite. Since $|\mathbf{N}|<|S|$, and $S \backslash \mathbf{N}$ is not left thick, $S$ is not strongly left thick. 
Since $\mathbf{N}$ is a right ideal of $S$, it is natural to ask whether every infinite left amenable semigroup, which is not strongly left thick, contains a right ideal of smaller cardinality. As far as we know, this question remains unanswered.

EXAMPLE 7.4. An infinite extremely left amenable semigroup $S$ with left cancellation, which is not uniformly left thick. As in Example $7.1 S$ is strongly left thick, but in this case $S$ does contain finite left thick subsets.

Let $S$ be any infinite set with the multiplication $s t=t$ for all $s, t \in S$. For each $s \in S$, the set $\{s\}=S s$ is left thick, so $S$ is not uniformly left thick. $S$ is extremely left amenable since for each $s \in S$ we can define $\mu_{s} \in M l(S)$ by $\mu_{s}(f)=f(s)$ for all $f \in m(S)$. Then each $\mu_{s}$ is also multiplicative.

8. Some final remarks. If $S$ is a uniformly left thick infinite left amenable semigroup, then the existence of a collection $\left\{D_{i}: i \in I\right\}$ of left thick subsets of $S$ with $\left|D_{i} \cap D_{j}\right|\langle|S|$ for $i \neq j$ would imply $\operatorname{dim}\langle M l(S)\rangle \geqslant|I|$. This follows from Proposition 3.1, since by choosing $\mu_{i} \in M l(S)$ with $\mu_{i}\left(\chi_{D_{1}}\right)=1$ for each $i \in I$, we have $\mu_{i}\left(\chi_{D_{j}}\right)=\mu_{i}\left(\chi_{\left(D_{i} \cap D_{j}\right)}\right)=0$ when $i \neq j$. Returning to the proof of Theorem 2.2, we see that such a collection can be found if and only if a collection $\left\{P_{i}: i \in I\right\}$ of cofinal subsets of $\Gamma=\{\alpha: \alpha<\omega\}$ can be found such that $\left|P_{i} \cap P_{j}\right|<|\Gamma|$ if $i \neq j$. When $S$ (and hence $\Gamma$ ) is countably infinite, such a collection can be found with $|I|=2^{\aleph_{0}}=\sqrt{ }$ (see for instance Chou [1, p. 781]).

This leads one to hope that when $\Gamma$ is infinite, one could always find such a collection with $|I|=2^{|\Gamma|}$. However, when $\omega$ is the first uncountable ordinal, the existence of such a collection is independent of the usual axioms of set theory $\left({ }^{2}\right)$. Thus when $S$ is uncountable, this technique cannot be used to obtain a better lower bound for $\operatorname{dim}\langle M l(S)\rangle$.

A further example of the limitations of using collections of left thick subsets to determine the exact size of $\operatorname{dim}\langle M l(S)\rangle$ is seen by considering $\operatorname{dim}\langle M l(G)\rangle$, where $G$ is an infinite amenable group. Using a technique of Kakutani and Oxtoby (see [11, pp. 215-225]), Chou has shown that $\operatorname{dim}\langle M l(G)\rangle=2^{2^{|G|}}$ in [3]. Since $G$ contains only $2^{|G|}$ distinct subsets, we cannot possibly obtain this result through the use of collections of left thick subsets.

\section{REFERENCES}

1. Ching Chou, Minimal sets and ergodic measures for $\beta \mathbf{N} \backslash N$, Illinois J. Math. 13 (1969), 777-788. MR 40 \#2814.

2. $\longrightarrow$ On the size of the set of left invariant means on a semigroup, Proc. Amer. Math. Soc. 23 (1969), 199-205. MR 40 \#110.

(2)This result was brought to my attention by R. Sirois-Dumais. It is stated in a preprint "Combinatorics" by K. Kunen. He has sent me the proof in a letter, mentioning that it was first obtained by Baumgartner in his Ph.D. thesis. 
3. The exact cardinality of the set of invariant means on a group, Proc. Amer. Math. Soc. 55 (1976), 103-106.

4. P. Civin and B. Yood, The second conjugate space of a Banach algebra as an algebra, Pacific J. Math. 11 (1961), 847-870. MR 26 \#622.

5. M. M. Day, Amenable semigroups, Illinois J. Math. 1 (1957), 509-544. MR 19, 1067.

6. L. R. Fairchild, Extreme invariant means without minimal support, Trans. Amer. Math. Soc. 172 (1972), 83-93. MR 46 \#7799.

7. E. Granirer, On amenable semigroups with a finite-dimensional set of invariant means. I, II, Illinois J. Math. 7 (1963), 32-48; ibid. 7 (1963), 49-58. MR 26 \# 1744, \# 1745.

8. A theorem on amenable semigroups, Trans. Amer. Math. Soc. 111 (1964), 367-379. MR $29 \# 3870$.

9. Extremely amenable semigroups, Math. Scand. 17 (1965), 177-197. MR 33 \#5760. 10. E. Granirer and M. Rajagopalan, $A$ note on the radical of the second conjugate algebra of $a$ semigroup algebra, Math. Scand. 15 (1964), 163-166. MR 32 \#2924.

11. E. Hewitt and K. Ross, Abstract harmonic analysis, Vol. 1, Academic Press, New York; Springer-Verlag, Berlin, 1963. MR 28 \# 158.

12. I. S. Luthar, Uniqueness of the invariant mean on an abelian semigroup, Illinois J. Math. 3 (1959), 28-44. MR 21 \#2184.

13. Theodore Mitchell, Constant functions and left invariant means on semigroups, Trans. Amer. Math. Soc. 119 (1965), 244-261. MR 33 \#1743.

Department of Mathematics, University of Alberta, Edmonton, Alberta, Canada T6G $2 \mathrm{Gl}$ 